\title{
Elaboración y validación de materiales para la formación del profesorado de Educación Primaria, con el objetivo de que sus alumnos aprendan la competencia de Pensamiento Computacional
}

\author{
Constanza Rubio Michavilaa ${ }^{a}$ Kilian González Suárez ${ }^{\mathrm{b}}$ y María Olga Escandell \\ Bermúdez $^{\mathrm{c}}$ \\ aUniversitat Politècnica de València, crubiom@fis.upv.es, bUniversidad de Las Palmas de Gran \\ Canaria, kilian57@gmail.com, 'Universidad de Las Palmas de Gran Canaria, \\ mariaolga.escandell@ulpgc.es
}

\begin{abstract}
Programming and Robotics are some of the disciplines that have entered the classrooms with the aim of developing one of the competencies that are considered most relevant in today's education: Computational Thinking. Computational Thinking helps to structure the minds of the students, providing the necessary tools to solve problems in a precise and orderly manner. This fact has motivated the creation and validation of a proposal for learning materials which would allow to improve the way in which children develop that competence. The greatest challenge would be to train future teachers so that they are able to encourage these skills in students.

To validate the learning materials, an exploratory research was carried out using a Delphi method based on two rounds in which 10 experts from different disciplines participated, all of them with a strong technological component. The experts completed a valuation questionnaire with the objective of analyzing the strengths and weaknesses of the proposal, taking as a reference the contents, the methodology, the resources and the evaluation system. Finally, after these assessments were carried out, a consensus was reached, validating the proposal and preparing it to be applied in a real context.
\end{abstract}

Keywords: competences, computational thinking, constructionism, problembased learning, project-based learning, training, methodology, primary education.

\footnotetext{
Resumen

La programación y la robótica son algunas de las disciplinas que han invadido las aulas con el objetivo de desarrollar una de las competencias consideradas de mayor relevancia en la educación de hoy día: el Pensamiento Computacional. El Pensamiento Computacional ayuda a estructurar la mente, aportando las herramientas necesarias para resolver problemas de un modo preciso y ordenado. Este hecho ha motivado la creación y validación de una propuesta de materiales de aprendizaje que permitan perfeccionar el modo en el que los niños mejoran dicha competencia. El gran reto se encuentra en
} 
Elaboración y validación de materiales para la formación del profesorado de Educación Primaria, con el objetivo de que sus alumnos aprendan la competencia de Pensamiento Computacional

formar y acompañar a los futuros profesores para que estos sean capaces de fomentar dichas habilidades en los alumnos.

Para validar los materiales, se realizó una investigación exploratoria que empleó un método Delphi basado en dos rondas en las que participaron 10 expertos de diferentes disciplinas, todos ellos con una fuerte componente tecnológica. Los expertos completaron un cuestionario de valoración para analizar los puntos fuertes y puntos débiles de la propuesta, tomando como referencia los contenidos, la metodología, los recursos y el sistema de evaluación. Finalmente, tras dichas valoraciones se alcanzó un consenso que validó la propuesta, quedando lista para ser aplicada en un contexto real.

Palabras clave: competencias, pensamiento computacional, construccionismo, aprendizaje basado en problemas, aprendizaje basado en proyectos, formación, metodología, educación primaria.

\section{Introducción}

La humanidad vive inmersa en una transformación que radica en gran parte en el hecho digital; un hecho que todavía se encuentra en sus comienzos. Esta situación afecta a todos, no importa la edad, la cultura, el entorno o la política. Para adaptarse a este nuevo contexto, la sociedad debe educarse y formarse en base a un conjunto de competencias, que permitan afrontar un futuro volátil, incierto, complejo y ambiguo que normalmente es conocido como VUCA.

Entre las competencias vinculadas al hecho digital, a nivel macro se encuentra el Pensamiento Computacional (PC). La investigación realizada se centra en esta competencia de orden superior, ya que permite trabajar con niños, adolescentes y jóvenes, mediante el uso de recursos abiertos y motivadores que siguen pedagogías constructivistas y construccionistas, y según algunos conectivistas.

S. Papert (1991) es considerado uno de los pioneros del Pensamiento Computacional y además es autor de la teoría construccionista, inspirada en el constructivismo de Piaget, con el que trabajó en Suiza. Según Ackerman (2001), los dos definen la inteligencia como la capacidad de adaptación, la capacidad para mantener el equilibrio entre la estabilidad y el cambio. A su vez, Jeannette M. Wing (2006) es quien utilizó por primera vez la denominación actual para el PC, definiéndolo como «la forma en la que los seres humanos resuelven los problemas complejos, incorporando una serie de procesos mentales que la ciencia informática, en cierto modo, utiliza».

Desarrollar el Pensamiento Computacional es más que aprender a codificar y programar, aunque estas habilidades se pueden utilizar para perfeccionar dicho tipo de pensamiento. El PC implica enseñar a pensar, a abordar problemas y resolverlos, desarrollando la metacognición, que es básica para aprender a aprender.

Integrar el aprendizaje del PC a nivel formal puede seguir distintos modelos, ya sea incluyendo una o varias herramientas específicas dentro de una asignatura ya existente,

(cc) EY-NC-ND 2018, Universitat Politècnica de València

Congreso IN-RED (2018) 
diseñando un nueva asignatura para sumarla al currículo actual o trabajándolo de manera transversal en todo el plan de estudios. Es un área con bastantes incógnitas y líneas abiertas, que se ha comenzado a plantear ya en las instituciones educativas de todo el mundo.

Uno de los primeros pasos a dar es el desarrollo de la propia competencia en el futuro profesorado que se encuentra en las facultades de ciencias de la educación. Para que estos sean capaces de guiar a otros es conveniente que primero cultiven la competencia por sí mismos. La investigación aborda este tema de un modo general, inclusivo y para todos, pudiéndose aplicar tanto en estudiantes universitarios como en sus futuros alumnos.

\section{Objetivos}

Con el desarrollo del PC en mente, es vital que el profesorado tenga a su disposición un banco de recursos, herramientas o software, para ser utilizado en la escuela, escogiendo cada docente aquellos con los que más cómodo se encuentre y que mejor se adapten a los objetivos de aprendizaje. La importancia de las innovaciones debe situarse en las pedagogías y los procesos pedagógicos, y no tanto en las tecnologías usadas.

El propósito de la investigación ha sido elaborar y validar un conjunto de herramientas para desarrollar el Pensamiento Computacional, mediante la propuesta metodológica que tenga en cuenta las peculiaridades del mismo y que mejor se adapte. Entre dichas propuestas metodológicas se valoraron el Aprendizaje Basado en Problemas, el Aprendizaje Basado en Proyectos y una mezcla entre ambas, de modo que puedan ser utilizadas en un determinado nivel educativo formal, integrado o no en el plan de estudios, para desarrollar la competencia de Pensamiento Computacional a partir de la capacitación mediante la codificación (coding) aplicada a problemas reales del mundo físico. Es importante destacar que aunque el Aprendizaje Basado en Proyectos y el Aprendizaje Basado en Problemas comparten muchas características, se trata de dos enfoques diferentes. En el Aprendizaje Basado en Problemas prima el proceso seguido por los alumnos mientras que en el Aprendizaje Basado en Proyectos prima el producto final.

Cuando se hace referencia al mundo físico, es para recalcar la importancia de que el problema esté vinculado al mundo real de las personas, que se encuentran a cargo de la búsqueda de una solución. Todo el conocimiento y el trabajo debe estar enfocado hacia su traslado desde el mundo real al mundo virtual, para terminar volviendo al mundo real (que es tangible) con la solución, sin descuidar en ningún momento que el alumnado debe ser consciente de este proceso.

Reformulando el propósito a modo de pregunta general, se definiría la siguiente cuestión: ¿Cómo es posible estudiar adecuadamente el desarrollo, entrenamiento y asimilación de dicha competencia, mediante el uso de herramientas propias del Pensamiento Computacional?

J. M. Wing (2006) resalta que el PC es una manera ordenada de pensar, de reflexionar sobre los diferentes problemas que se puedan presentar a lo largo de la vida, tanto en lo personal (individual y colectivo) como en lo profesional. El PC ayuda a estructurar y gestionar la gran 
Elaboración y validación de materiales para la formación del profesorado de Educación Primaria, con el objetivo de que sus alumnos aprendan la competencia de Pensamiento Computacional

cantidad de información que se recibe cada día. Este proceso mental es posible gracias a las tres fases del PC, que se pueden simplificar del siguiente modo:

- Descomposición: resolver un problema dividiéndolo en partes más pequeñas.

- Reconocimiento de patrones: encontrar la relación jerárquica, analizar los datos.

- Diseño algorítmico: obtener soluciones, siguiendo una serie ordenada de pasos.

Las tres fases anteriores se encuentran implícitas en la propuesta generada para abordar el Pensamiento Computacional desde una asignatura relacionada con las TICs. Por lo tanto, será necesario el uso de dispositivos tecnológicos. En este caso, se requiere de placas y componentes de electrónica educativa además de ordenadores de sobremesa y/o portátiles, que permitan ejecutar el software controlador Snap! Dicho software permite programar de manera visual mediante el uso de bloques, que representan instrucciones y que son interconectados para llevar a cabo determinadas acciones. A este conjunto de acciones que resuelven un problema se le llama algoritmo.

Por otra parte, la profesora M. Romero (2017) ha desarrollado el modelo \#5c21, en el que se refuerza la existencia de una relación bidireccional entre el Pensamiento Computacional y otras competencias que se consideran esenciales para el siglo XXI, haciendo referencia sobre todo a las 4C's (creatividad, comunicación, colaboración, y pensamiento crítico). Quiere decir esto que mediante el desarrollo del PC también se estarían desarrollando las 4C's, y de igual modo sucedería a la inversa.

\section{Desarrollo de la innovación}

Para llevar a cabo la validación de los materiales de aprendizaje desarrollados se ha utilizado el método Delphi, una técnica cualitativa aplicable en las investigaciones exploratorias, definidas por Fernández Alarcón (2006). La técnica Delphi utiliza cuestionarios para establecer un consenso entre un grupo de expertos que son guiados por un moderador. La información no es recogida a partir de un conjunto de casos reales, sino a través de la opinión de un conjunto de personas. Esta técnica está formada por tres fases: el diseño del cuestionario, la selección de los expertos y el envío del cuestionario con su posterior proceso iterativo de retroalimentación. Es importante destacar que en esta metodología el diseño del cuestionario debe ser lo más riguroso y completo posible, aunque las preguntas pueden ser abiertas y/o cerradas, así como cuantitativas y/o cualitativas.

Según Cuhls (2003), el método Delphi es una encuesta desarrollada en dos o más rondas, en las que en cada una de ellas se incorporan a los materiales diseñados las sugerencias y orientaciones aportadas por los expertos en rondas previas. Esta investigación optó por utilizar el método Delphi convencional de dos rondas, ya que gracias a las herramientas de comunicación de las que se dispone en la actualidad, el proceso de obtención del feedback es mucho más sencillo, pudiendo contactar con los expertos mediante correo electrónico y, en los casos en los que no fue posible, de forma presencial. Según Dalkey (1972), el anonimato de los sujetos que intervienen en el proceso Delphi es un punto a favor ya que este disminuye el efecto ruido, evitando así la influencia de las respuestas de los unos en los otros.

(c) EY-NC-ND 2018, Universitat Politècnica de València

Congreso IN-RED (2018) 
Para la selección de los expertos se propuso como criterio formar un grupo multidisciplinar, ya sea por su formación inicial o por su dedicación e intereses. Al abordar el diseño de los materiales para el desarrollo del Pensamiento Computacional desde un área de trabajo principalmente tecnológica, se procuró que la totalidad de los expertos tuviese relación con el hecho digital y con su integración en la educación y formación.

De este modo, los expertos han sido personas de perfil tecnológico muy vinculado a la educación como docentes, investigadores, desarrolladores de software, profesionales de la innovación e inclusión social, formadores de formadores, expertos en aprendizaje basado en problemas y coordinadores de programas educativos.

A continuación, aparece un listado con el perfil de los 10 expertos que han participado en el análisis:

- 2 docentes de tecnología e informática pertenecientes a la ESO.

- 1 docente de ciclo medio y superior.

- 1 formador de formadores a nivel universitario.

- 1 experto en Aprendizaje Basado en Problemas.

- 1 experta en educación inclusiva.

- 1 director de área en empresa de desarrollo de software.

- 1 decano de facultad de Informática, Multimedia y Telecomunicaciones.

- 1 especialista en el desarrollo y aplicación de TIC con fines sociales.

- 1 responsable de formación y divulgación de cultura científica.

Las herramientas utilizadas para validar la propuesta se basaron fundamentalmente en un cuestionario estructurado en base a 4 preguntas de respuesta cerrada, a las que se les dio un mayor valor cualitativo, ya que incluían un apartado destinado a comentarios; y 3 cuestionarios de respuesta abierta, en los que los expertos pudieron aportar cuáles, según ellos, son las fortalezas y debilidades del planteamiento, añadiendo además un apartado final para que incluyesen nuevas ideas que contribuyeron a la mejora del mismo.

Las preguntas de carácter cerrado son evaluadas mediante una escala de tipo Likert, pudiendo seleccionar diez niveles de respuesta, donde 10 es la puntuación máxima y 1 la mínima. Las variables medidas guardaron relación con los contenidos, la metodología, los medios/recursos y la herramienta de evaluación. Una evaluación que de ser exclusivamente cuantitativa pasaría por alto muchos aspectos de relevancia; por ello la herramienta incluyó las dos vertientes, cualitativa y cuantitativa.

Las preguntas, de carácter cerrado y puntuadas de 1 a 10, fueron las siguientes:

- ¿Considera adecuados los contenidos del programa, para abordar el coding y la interacción con el mundo físico?

- ¿Considera adecuada la metodología empleada (metodología mixta entre Aprendizaje Basado en Problemas y Aprendizaje Basado en Proyectos)?

- ¿Considera adecuados los medios y recursos tecnológicos (y no tecnológicos) empleados?

- ¿Considera adecuado el sistema de evaluación del programa (la rúbrica)? 
Elaboración y validación de materiales para la formación del profesorado de Educación Primaria, con el objetivo de que sus alumnos aprendan la competencia de Pensamiento Computacional

Aunque la aplicación del método Delphi puede variar, la propuesta llevada a la práctica se considera como caso típico de dicho método. Los resultados de la ronda de valoraciones iniciales son resumidos para calcular posteriormente la media y la desviación típica. Posteriormente, esta información fue devuelta a los participantes (segunda ronda) para que revisaran las estimaciones iniciales. Normalmente, el proceso se continúa de forma iterativa hasta alcanzar un consenso. El consenso se estableció de manera objetiva tras alcanzar una nota media superior a 8 en cada uno de los aspectos evaluados.

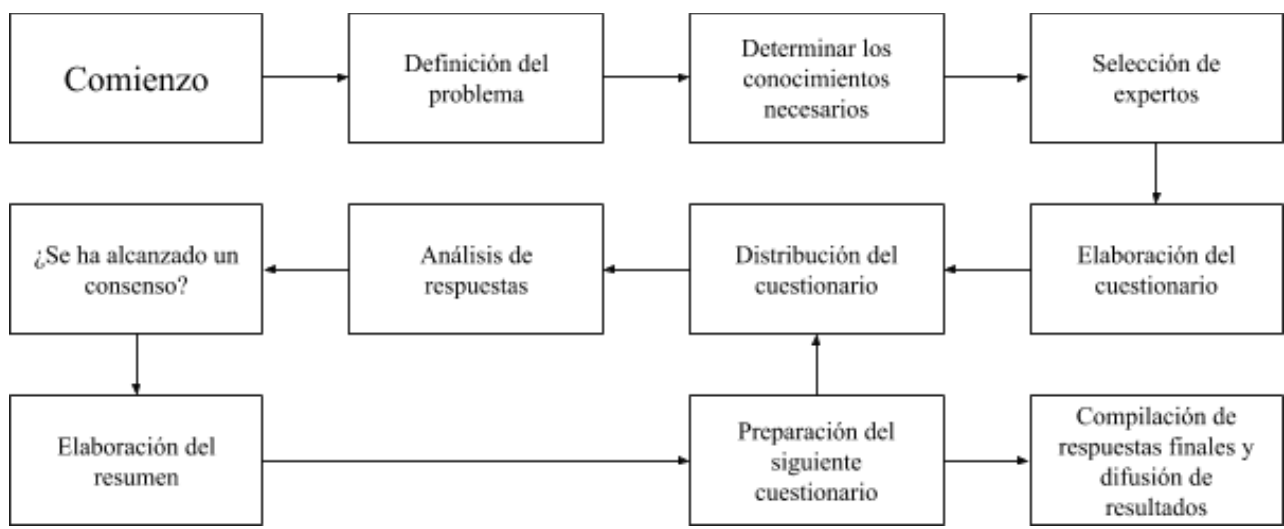

Fig. 1 Flujo estándar del desarrollo del método Delphi seguido.

\section{Resultados}

A continuación se presentan los resultados que se obtuvieron en cada ronda de valoración, analizándolos de manera independiente para iniciar la medidas de mejora propuestas por los expertos.

\subsection{Primera ronda de valoraciones}

Las preguntas de carácter cerrado arrojaron los siguientes valores cuantitativos:

Tabla 1. Medidas de tendencia central para la primera ronda de evaluación

\begin{tabular}{cccccc}
\hline & N & Mínimo & Máximo & Media & Desviación típica \\
\hline Contenidos & 10 & 7 & 10 & 8,80 & 0,919 \\
Metodología & 10 & 6 & 10 & 9,10 & 1,449 \\
Recursos & 10 & 8 & 10 & 9,40 & 0,843 \\
Evaluación & 9 & 5 & 10 & 8,22 & 1,716 \\
\hline
\end{tabular}

Se observó en los resultados que todas las calificaciones superaron el mínimo propuesto de 8 puntos, pero la segunda ronda de evaluación resultó igualmente necesaria para incorporar las opiniones de los expertos. Centrando la atención en las puntuaciones más bajas, destacan

(cc) EY-NC-ND 2018, Universitat Politècnica de València

Congreso IN-RED (2018) 
las variables correspondientes con los contenidos y la herramienta de evaluación. La desviación típica además demostró que existen irregularidades en las categorías correspondientes con la evaluación y la metodología.

Atendiendo a una explicación mucho más cualitativa de los datos estadísticos, gracias al complemento que supusieron las preguntas de carácter abierto, se resalta que, en el caso de los contenidos, las valoraciones fueron mayormente positivas, apuntando hacia la idoneidad de la propuesta en este aspecto y añadiendo como posible mejora la realización de un mayor esfuerzo en cuanto a la atención a la diversidad, asociando los contenidos con el currículo oficial y apoyando el aprendizaje mediante videos.

En el caso correspondiente con la metodología, es necesario destacar que la propuesta inicialmente solo se centró en el Aprendizaje Basado en Problemas, señalando los expertos que este es adecuado por considerar los procesos como eje principal del aprendizaje, pero la desviación típica observada señala también que algunos expertos no han estado del todo de acuerdo con el resto. Este desacuerdo fue atribuido a la preferencia que estos tenían por otro tipo de metodologías, concretamente el Aprendizaje Basado en Proyectos o de un enfoque mixto.

Respecto a los medios y recursos empleados en la propuesta educativa, todos los expertos remarcaron la conveniencia de los mismos, destacando además la importancia de generar una hoja de presupuesto que dotase al programa de mayor solidez y viabilidad. Además, a este aspecto se le sumó el entorno, la trascendencia de generar un ambiente propicio en el que llevar a cabo el proceso de enseñanza y aprendizaje.

Sin duda, la herramienta de evaluación escogida (una rúbrica) fue el aspecto en el que más estuvieron en desacuerdo los expertos, no tanto por el hecho de que se tratase de una rúbrica, sino debido al planteamiento y gradación de la misma. Estas opiniones dejaron entrever que la rúbrica requería de una reformulación.

\subsection{Segunda ronda de valoraciones}

En la segunda ronda, se obtuvieron los siguientes datos a partir de las preguntas de carácter cerrado:

Tabla 2. Medidas de tendencia central para la segunda ronda de evaluación

\begin{tabular}{cccccc}
\hline & N & Mínimo & Máximo & Media & Desviación típica \\
\hline Contenidos & 10 & 8 & 10 & 9,50 & 0,707 \\
Metodología & 10 & 8 & 10 & 9,60 & 0,843 \\
Recursos & 10 & 7 & 10 & 9,60 & 0,966 \\
Evaluación & 10 & 5 & 10 & 8,60 & 1,350 \\
\hline
\end{tabular}

En la segunda iteración de cuestionarios todas las puntuaciones sufrieron un incremento sustancial, superando el 9 en tres de los cuatro casos expuestos. El aspecto correspondiente 
Elaboración y validación de materiales para la formación del profesorado de Educación Primaria, con el objetivo de que sus alumnos aprendan la competencia de Pensamiento Computacional

a la evaluación mejoró, pero no consiguió situarse al mismo nivel que el resto de apartados; aún así la propuesta inicial se vió enriquecida a partir de las opiniones de los expertos. Los datos numéricos de la Tabla 2 se entienden desde las valoraciones cualitativas que los participantes realizaron, para cada una de las categorías contempladas, así como los puntos fuertes y los puntos débiles.

La variable relacionada con los contenidos mejoró en 0,70 puntos respecto de la fase inicial, resaltando en este apartado que los contenidos ganaron mucho valor al verse reordenados y complementados. La metodología mejoró en 0,50 puntos, destacándose la buena integración entre el Aprendizaje Basado en Problemas y el Aprendizaje Basado en Proyectos y permitiendo así que la propuesta fuese más flexible ante posibles restricciones o contratiempos. Los medios y recursos mejoraron en 0,20 puntos, ratificando lo que desde un primer momento se había planteado: son idóneos para el desarrollo del Pensamiento Computacional. Por otra parte, la valoración de la herramienta de evaluación mejoró notablemente, apuntando el panel de expertos que se trata de uno de los aspectos más difíciles en los que lograr el consenso, pues no todo el mundo comparte que un determinado método de evaluación con unas determinadas gradaciones puedan ser las más adecuadas. No obstante, esta variable experimentó una mejora de 0,38 puntos respecto de la propuesta inicial.

\section{Conclusiones}

A la vista de los resultados, se concluyó que la propuesta de materiales de aprendizaje para desarrollar el Pensamiento Computacional era válida, puesto que superó la calificación establecida como mínima para todos sus apartados; destacándose, entre otras cosas, la progresión y estructuración de los contenidos y las medidas de atención a la diversidad, que sustentan una curva de aprendizaje adecuada al perfil de cada estudiante. Por lo tanto es adecuada para el aprendizaje de los profesores de educación primaria, en su formación inicial.

La metodología mixta entre Aprendizaje Basado en Proyectos y Aprendizaje Basado en Problemas satisface a la forma en la que se ejecutaría el proyecto que deben afrontar los alumnos. Esta unión de metodologías es lo suficientemente flexible como para adaptarse a casi cualquier contexto, sobre todo si se atiende a la temporalización y a la formación docente del profesorado. La inclusión de vídeos eleva notablemente la calidad de la idea, ofreciendo de este modo un recurso atractivo que motive al alumnado y mantenga el interés de este.

A partir de la investigación realizada se abren nuevas líneas de trabajo orientadas hacia la mejora de las herramientas de evaluación, que deben ser reformuladas teniendo en cuenta cada contexto educativo concreto y creándose conjuntamente con el alumnado a partir del conocimiento del campo en el que van a ser aplicadas.

Queda pendiente la importante tarea de definir un proceso de cambio apropiado, que facilite la incorporación de estas propuestas y otras a las aulas ya existentes. El Pensamiento Computacional apunta a convertirse en una macrocompetencia esencial para el desarrollo del ser humano, teniendo en cuenta que estructuralmente será semejante y los contenidos diferentes, según la edad y capacidades de los aprendices.

(c) EY-NC-ND 2018, Universitat Politècnica de València

Congreso IN-RED (2018) 


\section{Referencias}

ACKERMANN, E. (2001). "Constructivisme et Constructionisme: Quelle Différence" en Constructivisms: Usages et Perspectives en Education. Geneva: Service de la recherche en education/Cahier 8/ September 01, Vol. 1 et 2, pp. 85-94.

CUHLS, K. (2003). "Development and perspectives of foresight in Germany" en Technikfolgenabschätzung, 2003. Vol. 12, n. 2, pp. 20-28.

DALKEY, N. C. (1972). Studies in the quality of life; delphi and decision-making. Lexington, USA: Lexington Books.

FERNÁNDEZ ALARCÓN, V. (2006). “Introducción a la investigación en ciencias sociales.” Working Paper del Departament d'Organització d'empreses de la Universitat Politècnica de Catalunya. <https://upcommons.upc.edu/bitstream/handle/2117/501/Introducci\%C3\%B3n\%20a\%201a $\% 20$ investigaci\%C3\%B3n\%20en\%20ciencias\%20sociales.pdf> [Consulta: 8 abril de 2017].

GONZÁLEZ SUÁREZ, K. (2017). Aprendizaje de competencias para el siglo XXI, mediante el desarrollo del pensamiento computacional, en alumnos de primaria: un caso piloto en Canarias. Las Palmas de Gran Canaria: Servicio de Publicaciones ULPGC. No listada.

PAPERT, S. y HAREL, I. (1993). Constructionism. Ablex Publishing Corporation, 1991.

ROMERO, M. (2017). “\#5c21 5 key skills for 21st century education” en \#CoCreaTIC, 27 de julio. <https://margaridaromero.wordpress.com/2016/07/28/5c21-5-key-skills-for-21st-century-

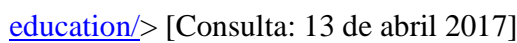

WING, J.M. (2006). "Computational thinking represents a universally applicable attitude and skill set everyone, not just computer scientists, would be eager to learn and use" en Communications of the ACM, 49, Vol. 49 Issue 3, March 2006, pp. 33-35. 